\title{
Fitting Zodiacal Models
}

\author{
Edward L. Wright \\ Institute for Advanced Study, Princeton NJ 08540 and UCLA \\ Astronomy, PO Box 951562, Los Angeles CA 90095-1562, USA
}

\begin{abstract}
Models of the zodiacal light are necessary to convert measured data taken from low Earth orbit into the radiation field outside the Solar System. The uncertainty in these models dominates the overall uncertainty in determining the extragalactic background light for wavelengths $\lambda<100 \mu \mathrm{m}$.
\end{abstract}

\section{Introduction}

The interplanetary dust particles responsible for the visible zodiacal light absorb most of the solar radiation that falls on them, and reradiate it in the infrared. Thus, a large part of the total infrared sky brightness from space is due to the zodiacal dust cloud. Modeling and removing this zodiacal foreground is an important part of the analysis of data from any space-based infrared experiment. The IRAS data have been fit both to physical three-dimensional models of the zodiacal cloud (Good et al.1986) and to phenomenological models of the variation with ecliptic latitude $\beta$ (Vrtilek \& Hauser 1995). The DIRBE data from $C O B E$ have been fit to 3-D models by Kelsall et al. (1998) and Wright et al. (1998), and for all of these fits the residuals are dominated by systematic errors in the 12 and $25 \mu \mathrm{m}$ bands where the signal-to-noise ratio on the zodiacal emission is high.

The fitting procedure used by Wright (1998) and Kelsall et al. (1998) allows for an arbitrary galactic plus extragalactic signal, $I_{c}$, at each pixel, but this arbitrary flux must be constant in time. All of the time variation of the observed signal, $I_{o b s}$, is assumed to be due to the changing line of sight through the zodiacal cloud as the Earth orbits around the Sun. Thus, the zodiacal model, $Z$, fit to the data is

$$
I_{o b s}(\lambda, l, b, t)=Z(\lambda, l, b, t ; p)+I_{c}(\lambda, l, b),
$$

where $\lambda$ is the wavelength, $l$ and $b$ are position coordinates, and $t$ is the epoch. The parameters of the model are the parameters, $p$, of the zodiacal light model plus the values $I_{c}$ - one value for each band and observed spot on the sky. There are thus a very large number of parameters in the model, but most of them are in $I_{c}$ and can be found directly because they are linear parameters. There are 11 other linear parameters in the Wright models which are the scattering efficiencies in bands 1-3 $(1.25-3.5 \mu \mathrm{m})$ and the emission efficiencies in bands $3-10(3.5-240$ $\mu \mathrm{m})$. 

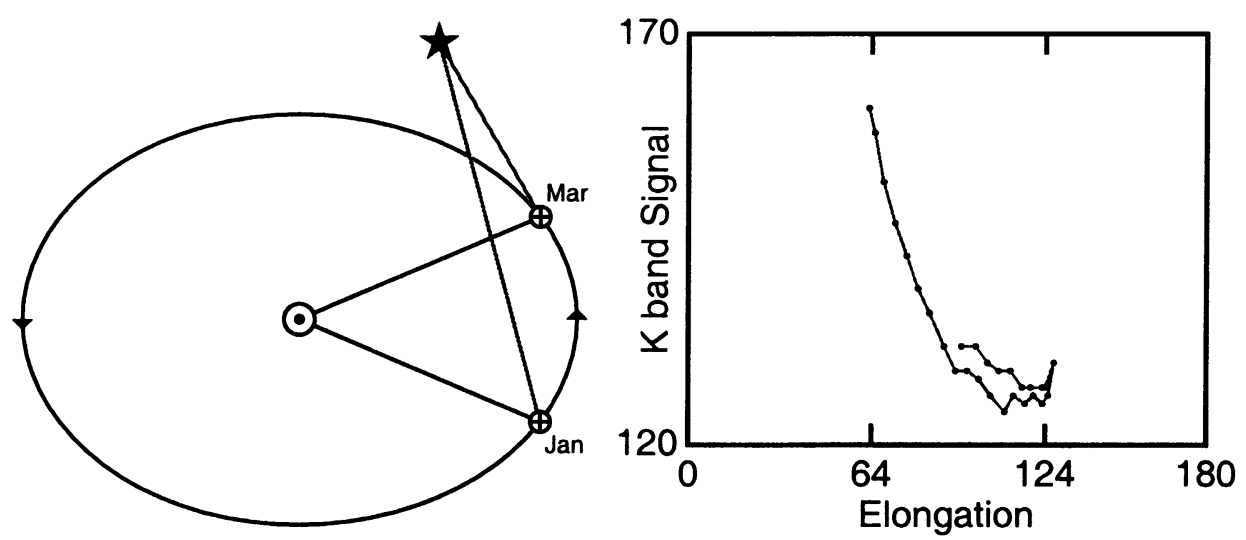

Figure 1. Left: Looking at the same position at different times of year gives different signals because of the different lines of sight through the interplanetary dust cloud. Right: The actual $2.2 \mu \mathrm{m}$ signal $v s$. angle between the line of sight (to the Gorjian, Wright \& Chary (2000) "dark spot") and the Sun.

I call this minimal assumption that the extrasolar system signal is independent of time the "weak no zodi principle". For a spherical shell of dust at radius $R>1 \mathrm{AU}$, the RMS time variability is only $\approx\left(4 / R^{2}\right) \%$ of the total intensity, which is a very weak signal indeed.

In order to save time, the model is only adjusted using a set of normal points corresponding to a set of spots on the sky. The main set of points were selected by picking points with very small gradients in $2^{\circ}$ patches both at $3.5 \mu \mathrm{m}$ and $100 \mu \mathrm{m}$. This selection reduces the "flicker noise" associated with bright sources which are sometimes in and sometimes out of the beam even while the beam center is always within a given pixel; and it eliminates points near the galactic plane or bright stars or bright cirrus clouds. There are 399 of these patches. An added set of points near the ecliptic but away from the Galactic plane are added when the parameters of the zodiacal bands are being determined. Finally, $10995^{\circ}$ patches at $|b|>20^{\circ}$ are used only for the 140 and $240 \mu \mathrm{m}$ bands, where the signal-to-noise ratio in the data is small.

In this paper I try more powerful assumptions about the celestial signal, and see how much leverage these give in fixing the zodiacal light. The first step in making assumptions about the sky is to assume that the $25 \mu \mathrm{m}$ sky at high $|b|$ is isotropic. This is a very reasonable assumption given the ratio of zodiacal to extrasolar system signals in this band. This is equivalent to reducing the 399 separate parameters in $I_{c}(25 \mu \mathrm{m}, l, b)$ to a single parameter $I_{c}(25 \mu \mathrm{m})$. I call this the "strong no zodi principle".

The next step in making assumptions about the sky is to assume that the extrasolar system high $|b|$ flux is zero. Since the zodiacal emission is 10 's of $\mathrm{MJy} / \mathrm{sr}$ while likely extragalactic backgrounds are only 10 's of $\mathrm{kJy} / \mathrm{sr}$, this assumption may be useful, but it is definitely a dangerous assumption to make when trying to find an isotropic extragalactic background. However, the fact that the model uses independent emissivities in each band, instead of assuming 
a smooth emissivity vs. wavelength law, means that the zodiacal model in the windows at 3.5 and $240 \mu \mathrm{m}$ is somewhat isolated from the assumptions made at $25 \mu \mathrm{m}$. Forcing the $25 \mu \mathrm{m}$ fit to a zero extrasolar system signal changes the shape of the cloud, and then this different shape leads to a different magnitude of the 3.5 or $240 \mu \mathrm{m}$ zodiacal flux. I call this assumption the "very strong no zodi principle".

Two models have been converged to the DIRBE Pass 3B data using all three fitting approaches. One is a "Good" model of the type used by Good et al. (1986). The other model is "FIZZ", a fairly elaborate physical model described in Wright (1998).

Table 1. Intensities in the Lockman Hole (MJy/sr)

\begin{tabular}{rrrrrrrrr}
\hline$\lambda[\mu \mathrm{m}]$ & GOOD1 & GOOD2 & GOOD3 & FIZZ1 & FIZZ2 & FIZZ3 & FIZZ3P & REALITY \\
\hline 1.25 & 0.1364 & 0.1407 & 0.1501 & 0.1696 & 0.1694 & 0.1806 & 0.1797 & 0.2228 \\
2.2 & 0.0942 & 0.0971 & 0.1037 & 0.1171 & 0.1170 & 0.1247 & 0.1240 & 0.1492 \\
3.5 & 0.0770 & 0.0788 & 0.0856 & 0.0897 & 0.0913 & 0.0976 & 0.0974 & 0.1149 \\
5 & 0.4287 & 0.4491 & 0.4699 & 0.4993 & 0.4954 & 0.5097 & 0.5088 & 0.5389 \\
12 & 13.4690 & 14.1695 & 14.7320 & 16.5817 & 16.2459 & 16.8580 & 16.8374 & 16.5239 \\
25 & 24.6018 & 27.2783 & 30.4484 & 29.2170 & 28.4249 & 30.2288 & 30.2234 & 30.0306 \\
60 & 6.8324 & 7.2153 & 7.4800 & 8.3259 & 8.0314 & 8.6145 & 8.6166 & 8.7382 \\
100 & 2.4155 & 2.5468 & 2.6346 & 3.0647 & 2.9380 & 3.1912 & 3.1932 & 4.1884 \\
140 & 1.1585 & 1.2219 & 1.3091 & 1.4817 & 1.4246 & 1.5838 & 1.5848 & 2.4480 \\
240 & 0.3571 & 0.3769 & 0.4028 & 0.4622 & 0.4414 & 0.4923 & 0.4927 & 0.9459 \\
\hline
\end{tabular}

The values of the zodiacal models, averaged over the actual observation times, for a set of pixels in the Lockman hole, are given in Table 1. GOOD1 and FIZZ1 use the "weak no zodi principle", GOOD2 and FIZZ2 the "strong no zodi principle", and GOOD3 and FIZZ3 the "very strong no zodi principle". The REALITY column gives the DIRBE data, i.e., total sky brightness, in the Lockman hole. The ratio of 240 to $25 \mu \mathrm{m}$ flux is fairly constant for the FIZZ models, ranging from 0.0155 to 0.0163 . This suggests that a simple calculation that assigns an uncertainty to the $25 \mu \mathrm{m}$ background can be used to determine the uncertainty of the zodiacal light model in the $240 \mu \mathrm{m}$ band. Thus, simple fitting procedures such as the one used by Schlegel, Finkbeiner \& Davis (1998) should be adequate for determining the $240 \mu \mathrm{m}$ background. At $3.5 \mu \mathrm{m}$ the situation is more ambiguous because of the scattered radiation, which amounts to about $50 \%$ of the total flux in this window. Changes in the assumed phase function for scattering have no effect whatsoever at $25 \mu \mathrm{m}$ but can have significant effects at $3.5 \mu \mathrm{m}$. Thus the model changes produced by the three different fitting procedures considered here do not adequately span the total range of possible models and could underestimate the systematic errors in the zodiacal flux in the short wavelength window. Even so, letting $\ln \Phi$ (where $\Phi$ is the scattering phase function) be a quartic (FIZZ3P in Table 1) instead of quadratic polynomial in $\mu$, the cosine of the scattering angle, produces only a $0.2 \mathrm{kJy} / \mathrm{sr}$ change in the zodiacal light model at $3.5 \mu \mathrm{m}$ in the Lockman hole. 


\section{References}

Good, J. C., Gautier, T. N., \& Hauser, M. G. 1986, AdvSpRes, 6 no. 7, 83 Gorjian, V., Wright, E., \& Chary, R. 2000, ApJ, 536, 550

Kelsall, T., et al. 1998, ApJ, 508, 44

Schlegel, D., Finkbeiner, D., \& Davis, M. 1998, ApJ, 500, 525

Vrtilek, J. M., \& Hauser, M. G. 1995, ApJ, 455, 677

Wright, E. 1998, ApJ, 496, 1 